\title{
Fabrication and characterisation of substrate-free PZT thick films
}

\author{
Dazhi Wang ${ }^{\mathrm{a}, \mathrm{b}, *}$, Peng Shi ${ }^{\mathrm{a}}$, Xuemu $\mathrm{Li}^{\mathrm{c}}$, Peng Zhou ${ }^{\mathrm{a}}$, Kuipeng Zhao ${ }^{\mathrm{a}}$, Yunlong Wei ${ }^{\mathrm{a}}$, \\ Chongyang Jianga ${ }^{\mathrm{a}}$, Junsheng Liang ${ }^{\mathrm{a}, \mathrm{b}}$, R.A. Dorey ${ }^{\mathrm{d}}$
}

${ }^{a}$ Key Laboratory for Micro/Nano Technology and System of Liaoning Province, Dalian University of Technology, Dalian, 116024, China

${ }^{\mathrm{b}}$ Key Laboratory for Precision and Non-traditional Machining Technology of Ministry of Education, Dalian University of Technology, Dalian, 116024, China

${ }^{c}$ Key Laboratory of High Efficiency and Clean Mechanical Manufacture of MOE, School of Mechanical Engineering, Shandong University, Jinan 250061, China

${ }^{\mathrm{d}}$ Department of Mechanical Engineering Sciences, University of Surrey, Surrey GU2 $7 \mathrm{XH}, \mathrm{UK}$

\begin{abstract}
This paper reports the preparation of dense and substrate-free PZT thick films. Electrohydrodynamic jet deposition and sol infiltration were utilized to produce dense PZT thick film, then wet chemical etching was employed to successfully remove the silicon substrate. Subsequently, a pure PZT thick film having a thickness of $14 \mu \mathrm{m}$ without substrate was produced. The piezoresponse force microscopy technique was used to examine the piezoelectric constant $\left(d_{33, f}\right)$, it was found that the $d_{33}$ was increased from $71 \mathrm{pm} \mathrm{V}^{-1}$ to $140 \mathrm{pm} \mathrm{V}^{-1}$, having a double increase. It was also observed that the remnant polarization $\left(P_{r}\right)$ and relative permittivity $\left(\varepsilon_{r}\right)$ of PZT film were distinctly improved after the removal of silicon substrate. The experimental result shows that the substrate clamping had great effects on the electrical properties of PZT films and its effect value was evaluated. In addition, the systematic theoretical analysis of the substrate clamping on film was deeply studied. The theoretical analysis agrees well with the experiment results, which can be used to estimate the effect value caused by the substrate clamping.
\end{abstract}

Keywords: Electrohydrodynamic jet deposition; Sol infiltration; Lift-off; Substrate-free PZT thick films

*Corresponding author. Email: d.wang@dlut.edu.cn; Tel.: +86(411)84707170-2171; Fax: +86(411)84707940 


\section{Introduction}

Lead zirconate titanate (PZT) is widely used for piezoelectric applications such as transducers and actuators due to its high electromechanical coupling coefficient, relative permittivity and piezoelectric constant [1]. For applications in different fields, PZT films with various thickness ranges have been fabricated. Recently, there has been increasing interest in PZT thick films ranging from $10 \mu \mathrm{m}$ to $100 \mu \mathrm{m}$, which combine the performance approaching those of bulk ceramics with the easy system integration and miniature size scale. PZT thick films at this micro-scale are favourable for the micro-electromechanical systems (MEMS) applications such as micro-pumps [2], highfrequency ultrasonic sensors [3] and micro-energy harvesters [4] owing to its large driving power, wide frequency range and high sensitivity.

The production of PZT thick films with high quality is the key issue to achieve the expected performance of these MEMS applications. In recent years, various techniques have been investigated for fabricating PZT thick film. Screen printing technology was investigated to fabricate PZT thick film where the PZT paste was printed on the screen stencil for the formation of patterned PZT thick film [5]. Aerosol deposition (AD) has been studied to fabricate PZT thick films by consolidation of submicron-ceramic particles on substrate at room temperature [6]. Composite film (ComFi) technique utilizes the combination of PZT powder and sol-gel processing to produce PZT thick film $[7,8]$, which was introduced to fabricate PZT thick film with the thickness of tens of micrometres.

In our previous work, electrohydrodynamic jet (E-Jet) deposition technology combined with composite PZT powder/sol slurry has been employed to fabricate PZT thick film $[9,10]$ and micro-scale structures $[11,12]$. E-Jet utilizes mechanical and electrical 
forces to create an ink jet and further break it up into droplets, which has the advantages of high controllability, high density and fast speed for producing PZT thick films. In addition to preparing PZT thick films, E-Jet is also utilized for high-resolution preparing PZT thin films with the thickness of a few micrometres [13]. It was reported that the PZT thin film presented uniform and accurate feature. Recently, templateassisted electrohydrodynamic atomisation (TAEA) technique was developed to prepare well-defined surface topography with high resolution for biomedical applications by using ground electrode configurations and templates [14]. Owing to the same nature with E-Jet, TAEA can be reasonably adapted to pattern PZT thick film structures directly by using suitable PZT slurry mentioned above without the additional photolithography and etching process [12], which can simplify the fabrication process. Besides the PZT fabrication, E-Jet has exhibited abundant applications potential in the fields of biology, energy and food. Ghanbar et al. [15] reported their work on production of poly (glycolic-co-lactic acid) (PLGA) microspheres within the diameter range of 150-300 $\mu \mathrm{m}$ using E-Jet. Park et al. [16] presented the fabrication of efficient and flexible organic solar cells (OSCs) with an anode by E-Jet deposition of silver nanowires (Ag NWs). Luo et al. [17] produced near-monodisperse chocolate particles using E-Jet and manipulated the morphology of the prepared particles by varying process parameters.

Han et al. produced PZT thick films on silicon substrate with coated platinum successfully using aerosol deposition and studied the effects of film thickness [6] and top electrode diameter [18] on the piezoelectric properties. Kerkache et al. [19] investigated the dielectric and ferroelectric properties of the RF sputtered ITO/PZT/Pt structure on $\mathrm{SiO}_{2} / \mathrm{Si}$ substrates. Kuscer et al. [20] reported their results on the piezoelectric properties of ink-jet-printed PZT thick films on alumina substrate. 
However, in these works, the PZT thick films were deposited on a certain substrate, which could induce the effect of substrate clamping, resulting in the difficulty of domain wall motion and the reduction of film properties [21].

In this work, substrate-free PZT thick films were fabricated by E-Jet deposition and wet chemical etching technique, then their electrical properties were evaluated. The composite PZT powder/sol slurry was deposited on silicon substrate by the E-Jet deposition technology, and the PZT sol infiltration process was carried out for the purpose of increasing the density and electrical properties of deposited films. Then the sol infiltrated PZT thick films were removed from the silicon substrates through a wet chemical etching technology to examine the electrical properties without substrate clamping effect. With the use of piezoresponse force microscopy (PFM) technique, the piezoelectric coefficients $\left(d_{33, f}\right)$ of E-Jet deposited thick films before and after the removal of substrate were examined.

\section{Experimental details}

\subsection{E-Jet deposition and sol infiltration of PZT thick films}

To prepare the PZT slurry, $10 \mathrm{ml}$ PZT sol, $10 \mathrm{~g}$ PZT powder (Pz26, Ferroperm Piezoceramics, MEGGITT, Denmark), $2 \mathrm{ml}$ glacial acetic acid (99.8 wt\%), $2.2 \mathrm{ml} 1$ propanol (99.7 wt\%) and $0.2 \mathrm{~g}$ dispersant KR 55 (Ken-React Lica 38, KenRich, USA) were mixed and then ball-milled for $50 \mathrm{~h}$. The PZT sol was prepared from the precursors lead (II) acetate trihydrate, zirconium (IV) propoxide (76 wt\% in 1-propanol) and titanium (IV) isopropoxide (99.99 wt\% purity). The detailed preparation routes were described in our previous work [11].

The E-Jet deposition apparatus is shown in Fig. 1, which mainly consists of a computer controlled $X-Y$ movement stage, a syringe pump (PHD ULTRA 70-3307, Harvard 
Apparatus, USA), a high voltage power supply (DWP-P502-50ACF3, Tianjin DongWen High Voltage Power Supply Co., Ltd, China) and an E-Jet deposition needle. The needle, with the outer/inner diameter of $0.7 / 0.2 \mathrm{~mm}$, was connected to the high voltage power supply and its inlet was connected to the syringe pump by a silicone rubber tube. A thin aluminium plate with an area of $130 \mathrm{~mm} \times 130 \mathrm{~mm}$ was placed on the movement stage directly acting as the ground electrode. The PZT slurry was pushed to the outlet of the needle due to the hydrodynamic force provided by the syringe pump. The high voltage power supply provided an electric field between the plate ground electrode and the needle. The flow rate of the slurry, the applied voltage and the distance between the silicon substrate and the needle were kept at $1.67 \times 10^{-10} \mathrm{~m}^{3} \mathrm{~s}^{-1}, \sim 4 \mathrm{kV}$ and $\sim 4 \mathrm{~mm}$, respectively, to form stable cone-jet mode. The substrate was raster scanned alternating between one layer in the $X$ direction and another layer in the $Y$ direction to deposit the film over a large area. In order to ensure a degree of overlap between the deposited materials, the distance between the two parallel deposition paths was kept at $1.4 \mathrm{~mm}$ and the moving speed of the substrate was set to $35 \mathrm{~mm} \mathrm{~s}^{-1}$.

To increase the density of E-Jet deposited film, sol infiltration process was conducted by spin coating ( $2000 \mathrm{rpm}$ for $30 \mathrm{~s}$ ). The film was sol infiltrated one layer after every two layers of the E-Jet deposition. This process was employed four times, and the PZT film with the thickness of $\sim 14 \mu \mathrm{m}$ was obtained on the silicon substrate with sputtered Ti/Pt (10/100 nm). Scanning electron microscope (JSM-6360LV, JEOL Ltd., Japan) was used to measure the thickness of E-Jet deposited PZT thick film. For the purpose of removing the organic components and reducing the internal stress, the PZT thick films were dried at $200{ }^{\circ} \mathrm{C}$ for $60 \mathrm{~s}$ and then pyrolysed at $350{ }^{\circ} \mathrm{C}$ for $60 \mathrm{~s}$ on a hotplate after every layer deposition and infiltration. The completed film was sintered at $720{ }^{\circ} \mathrm{C}$ in muffle furnace for $20 \mathrm{~min}$ to develop the perovskite structure. 


\subsection{Lift-off of PZT thick films}

In this work, the sintered PZT thick film was removed from the silicon substrate by placing it in the $\mathrm{KOH}$ solution with a concentration of $40 \mathrm{wt} \%$ at a temperature of $85^{\circ} \mathrm{C}$. After 30 min wet etching, the PZT thick film with the Ti/Pt electrode was separated from the silicon substrate. The fabrication procedures of the substrate-free PZT thick films are shown in Fig. 2.

\subsection{Electrical measurements of PZT thick films}

Prior to the electrical measurements, Pt/Ti electrodes with a diameter of $2 \mathrm{~mm}$ and a thickness of 200/30 nm were sputtered onto the surface of PZT thick film by magnetron sputtering apparatus (LAB.18, Kurt J.Lesker, USA). The ferroelectric hysteresis loops of PZT thick films were tested by an improved Sawyer-Tower circuit. The remnant polarization $\left(P_{r}\right)$ can be calculated from the ferroelectric hysteresis loop. The dielectric loss and relative permittivity $\left(\varepsilon_{r}\right)$ were measured by a LCR tester (ZL5, Shanghai Instrument Institute, China), the frequencies was varied from 1 to $100 \mathrm{kHz}$. With the use of high voltage PFM module, the piezoelectric coefficients $\left(d_{33, f}\right)$ were measured using PFM which was conducted by a scanning probe microscope (SPM) (MFP-3D, Asylum Research, USA). The measurement of the piezoelectric deformation of the thick film caused by an applied electric field is based on the principle of PFM technique [22]. In order to evaluate local piezoelectric responses, surface of the E-Jet deposited thick films with and without silicon substrate was scanned in the range of 1 to $10 \mathrm{~V}$ through applying an AC driving voltage via the conductive tip (Ultrasharp NSC18/Pt/AlBS, MicroMasch, Estonia). The amplitude-voltage butterfly loops and the phase-voltage hysteresis loops were acquired by detecting the piezoelectric vibration 
signal, and the piezoelectric coefficient $\mathrm{d}_{33}$ could be further evaluated through the Eq.

$d_{33}=\operatorname{Acos}(\varphi) V_{a c}^{-1}$

where $A$ is the difference between the maximum and the minimum values of the PFM amplitude, $\varphi$ is the PFM phase, and $V_{a c}$ is the amplitude of the AC modulation. In this work, the sample deformation can be described as Eq. (2), because the polarization direction was parallel to the applied electric field.

$A=d_{33} V_{a c}$

\section{Results and discussion}

\subsection{E-Jet deposition, sol infiltration and lift off of the thick films}

In this work, the E-Jet deposition technique was carried out to form PZT thick films using the composite PZT slurry. Although the crack-free PZT thick films were produced with the use of E-Jet deposition combined with PZT slurry, submicron-pores were also presented in the film (Fig. 3a). The internal stress around the pores could hinder the wall domain motion, thereby decreasing the electrical properties of the films [23]. PZT sol infiltration process was carried out on the E-Jet deposited PZT thick films to increase the density. Fig. 3a and $\mathbf{b}$ show the surface of PZT thick films with and without infiltration. In this work, $(2 \mathrm{C}+\mathrm{S})$ sol infiltration was employed to form dense PZT thick films. The $(2 \mathrm{C}+\mathrm{S})$ denoted the process of the composite slurry deposition and sol infiltration, which means the film was infiltrated one layer after every two layers of E-Jet deposition. It was shown from Fig. 3b that after $(2 \mathrm{C}+\mathrm{S})$ sol infiltration the amount of submicron-pores between the PZT particles (Fig. 3a) decreased obviously and the film represented smoother and denser surface. As a result, the electric properties 
of the film can be improved. It was shown from Fig. 3c that the PZT thick film was entirely removed from the silicon substrate without any damages, which indicates that wet etching technique is a suitable way for the lift-off of PZT thick films from the silicon substrate.

\subsection{Electrical characterisation of the PZT thick films}

Fig. 4 shows the ferroelectric hysteresis loops of E-Jet deposited PZT thick films with and without silicon substrate. The remnant polarization $\left(P_{r}\right)$ of PZT thick film without substrate $\left(29.6 \mu \mathrm{C} \mathrm{cm}^{-2}\right)$ was approximately double of that with substrate $\left(16.1 \mu \mathrm{C} \mathrm{cm}^{-}\right.$ ${ }^{2}$ ), which is due to the decrease of the residual stress after removing the silicon substrate. The stress induced by the substrate is known to cause the perovskite domains to orient along the lateral direction, which results in the decrease of the ferroelectric properties

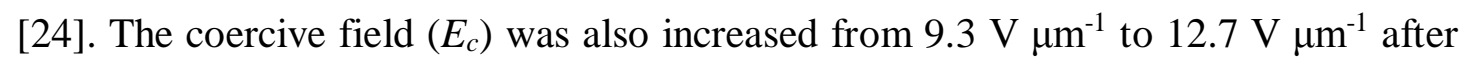
removing the substrate.

Fig. 5 and Fig. 6 show the relative permittivities $\left(\varepsilon_{r}\right)$ and dielectric losses of the E-Jet deposited PZT thick films with and without silicon substrate, respectively. It can be seen that the relative permittivity $\left(\varepsilon_{r}\right)$ was increased by the removal of the substrate in the frequency range of 1 to $100 \mathrm{kHz}$ and the dielectric losses were all less than 0.06 and did not change notably. The relative permittivity $\left(\varepsilon_{r}\right)$ of PZT thick film after removing the substrate was 433 at $50 \mathrm{kHz}$ and was higher than that with substrate of 400, which is due to the change of $90^{\circ}$ domain wall motion in the film. The polarization can be changed by the $90^{\circ}$ domain wall motion, which can contribute to the dielectric properties. It was reported that the $90^{\circ}$ domain wall motion would be severely limited by the residual stress, thus resulting in the lower dielectric constant [25]. The 
elimination of the substrate will result in a reduction of the residual stress, then encourage the $90^{\circ}$ domain wall motion and improve the dielectric properties.

The films were effectively polarized under the conductive AFM tip by applying a DC bias voltage sequence up to $90 \mathrm{~V}$ and $150 \mathrm{~V}$ via the AFM tip onto the PZT thick films with and without silicon substrate, respectively. Using an AC modulation voltage of 4 $\mathrm{V}$, the corresponding piezoresponse of films with and without substrate were measured. Fig. 7 and Fig. 8 show the obtained PFM phase-voltage hysteresis loops and amplitudevoltage butterfly loops of films with and without silicon substrate, respectively. Two different locations of films with and without substrate were selected to take the measurements. The piezoelectric coefficient $\left(d_{33}\right)$ of the E-Jet deposited thick film was evaluated by the following Eq. (3):

$$
d_{33}=A V_{a c}^{-1}
$$

The piezoelectric coefficients of the films with and without silicon substrate were measured to be $\sim 71 \mathrm{pm} \mathrm{V}^{-1}$ and $\sim 140 \mathrm{pm} \mathrm{V}^{-1}$, respectively. The stress induced by the substrate could limit the motion of the PZT thick film as it attempts to contract or expand under an external voltage, which is known to be the substrate clamping effect. Therefore, $d_{33}$ of the E-Jet deposited thick films has a double increase (from $\sim 71 \mathrm{pm} \mathrm{V}^{-}$ ${ }^{1}$ to $\sim 140 \mathrm{pm} \mathrm{V}^{-1}$ ) when the substrate was removed. The theoretical analysis [21] of the substrate clamping effect is described as follows.

Fig. 9 shows the notation of crystal axes with polarization direction on the PZT thick film. According to the piezoelectric constitutive equation, the strain of direction 1 and direction 3 and the electric displacement of direction 3 can be obtained by expending the tensor notation: 
$S_{1}=s_{11}^{\mathrm{E}} T_{1}+s_{12}^{\mathrm{E}} T_{2}+s_{13}^{\mathrm{E}} T_{3}+d_{31} E_{3}$

$S_{3}=s_{13}^{\mathrm{E}}\left(T_{1}+T_{2}\right)+s_{33}^{\mathrm{E}} T_{3}+d_{33} E_{3}$

$D_{3}=\varepsilon_{3}^{\mathrm{T}} E_{3}+d_{31}\left(T_{1}+T_{2}\right)+d_{33} T_{3}$

where $E, D, T$ and $S$ are the electric field $\left(\mathrm{V} \mathrm{m}^{-1}\right)$, electric displacement $\left(\mathrm{C} \mathrm{m}^{-2}\right)$, stress $\left(\mathrm{N} \mathrm{m}^{-2}\right)$ and strain, $\varepsilon, d$ and $s$ are the dielectric constant $\left(\mathrm{F} \mathrm{m}^{-1}\right)$, piezoelectric constant $\left(\mathrm{C} \mathrm{N}^{-1}\right)$ and compliance $\left(\mathrm{m}^{2} \mathrm{~N}^{-1}\right)$, respectively.

The direct effect concerning the amount of charge that the PZT film generated under an applied force is used to deduce the piezoelectric coefficient. Based on the assumption that the film is dominated by an isotropic substrate, the lateral strain in each direction is the same and therefore $S_{1}=S_{2}$ and the stress $T_{1}=T_{2}=T$ because of the substrate clamping effect. Based on the Hooke's law, $S_{1}$ and $S_{2}$ can be deduced as a function of $T_{3}:$

$S_{1}=S_{2}=-\left(\frac{v_{s u b}}{Y_{\text {sub }}}\right) T_{3}$

where $Y_{s u b}$ and $v_{s u b}$ are Young's modulus and Poisson's ratio of the substrate, respectively. Combining Eq. (4), (5) and (7) with the relation concerning the ratio of charge generated to the applied force and the strain, Eq. (8) can be obtained:

$$
-\left(\frac{v_{s u b}}{Y_{s u b}}\right) T_{3}=\left(s_{11}^{\mathrm{E}}+s_{12}^{\mathrm{E}}\right) T+s_{13}^{\mathrm{E}} T_{3}+d_{31} E_{3}
$$

According to the short circuit condition that $E_{3}=0$, Eq. (9) can be obtained by combining the assumptions about the direct effect with Eq. (6) and (8):

$$
\left(\frac{D_{3}}{T_{3}}\right)_{\mathrm{E}}=\left(\frac{2 d_{31} T+d_{33} T_{3}}{T_{3}}\right)_{\mathrm{E}}
$$


By rearranging Eq. (8) and substituting into Eq. (9), Eq. (10) can be obtained:

$$
\left(\frac{D_{3}}{T_{3}}\right)_{\mathrm{E}}=d_{33(s u b)}=d_{33}-2 d_{31}\left(\frac{v_{s u b} / Y_{s u b}+s_{13}^{\mathrm{E}}}{s_{11}^{\mathrm{E}}+s_{12}^{\mathrm{E}}}\right)
$$

where $d_{33(s u b)}$ and $d_{33}$ are the piezoelectric coefficients of the E-Jet deposited PZT thick films with and without substrate, respectively. The values of the Pz26 type materials were obtained from Meggitt [26]. The Young's modulus and Poisson's ratio values for silicon substrate are $160 \mathrm{GPa}$ and 0.22 , respectively. Eq. (11) can be obtained by substituting these values into Eq. (10).

$$
d_{33}=d_{33(s u b)}-1.31 d_{31}
$$

From the Meggitt data book, the typical values for $d_{31}$ is $-50 \mathrm{pm} \mathrm{V} \mathrm{V}^{-1}$. Before removing the substrate the piezoelectric coefficient of the E-Jet deposited PZT thick film is 71 $\mathrm{pm} \mathrm{V}^{-1}\left(d_{33(s u b)}\right)$. It can be calculated that the theoretical value for the piezoelectric coefficient of the deposited PZT thick film without silicon substrate is $136.5 \mathrm{pm} \mathrm{V}^{-1}$ $\left(d_{33}\right)$ by substituting $-50 \mathrm{pm} \mathrm{V}^{-1}\left(d_{31}\right)$ and $71 \mathrm{pm} \mathrm{V}^{-1}\left(d_{33(s u b)}\right)$ into Eq. (11), which is almost the same with the measured value $\left(140 \mathrm{pm} \mathrm{V}^{-1}\right)$. It can be observed that a double increase of the piezoelectric coefficient occurred on the E-Jet deposited PZT thick films after the removal of silicon substrate. This agree well with the experiment results and can give an estimation of the reduction value caused by the substrate clamping effect.

\section{Conclusions}

A series of fabrication processes for depositing PZT thick film and forming substratefree PZT thick film were demonstrated in this work. Dense PZT thick films were produced by the use of E-Jet deposition and sol infiltration. In order to exclude the influence of substrate clamping effect, the silicon substrate was then successfully 
removed through a wet chemical etching of $\mathrm{KOH}$ solution. Subsequently, a substratefree PZT thick film with a thickness of $14 \mu \mathrm{m}$ was obtained. After the removal of silicon substrate, the remnant polarization $\left(P_{r}\right)$ and relative permittivity $\left(\varepsilon_{r}\right)$ of PZT thick films were observed to be increased from $16.1 \mu \mathrm{C} \mathrm{cm}^{-2}$ to $29.6 \mu \mathrm{C} \mathrm{cm}^{-2}$ and from 400 to 433 , respectively, which was a significant improvement. In addition, the piezoelectric constant $\left(d_{33, f}\right)$ was examined using PFM technique, it was found that the $\mathrm{d}_{33}$ exhibited a double increase from $71 \mathrm{pm} \mathrm{V}^{-1}$ to $140 \mathrm{pm} \mathrm{V}^{-1}$ after removing the substrate. The experiment result shows that the electrical properties of PZT film were greatly affected by the substrate clamping. Furthermore, the systematic theoretical analysis of the substrate clamping on film was carried out, which agrees well with the experiment result and can be used to estimate the effect value caused by the substrate clamping. These processes demonstrated a promising way for preparing dense and substrate-free PZT thick film.

\section{Acknowledgements}

This research is supported by National Natural Science Foundation of China (No. 51475081, 51675085), Science Fund for Creative Research Groups of NSFC (51621064), the Fundamental Research Funds for the Central Universities (DUT18LAB17) and the Collaborative Innovation Center of Major Machine Manufacturing in Liaoning. 


\section{References}

[1] J.F. Scott, C.A.P. Dearaujo, Ferroelectric memories, Science 246 (1989) 1400-1405.

[2] P. Dhananchezhiyan, S.S. Hiremath, Optimization of multiple micro pumps to maximize the flow rate and minimize the flow pulsation, Procedia Technology 25 (2016) 1226-1233.

[3] M. Ito, N. Okada, M. Takabe, M. Otonari, D. Akai, K. Sawada, M. Ishida, High sensitivity ultrasonic sensor for hydrophone applications, using an epitaxial $\mathrm{Pb}(\mathrm{Zr}, \mathrm{Ti}) \mathrm{O}_{3}$ film grown on $\mathrm{SrRuO}_{3} / \mathrm{Pt} / \gamma-\mathrm{Al}_{2} \mathrm{O}_{3} / \mathrm{Si}$, Sens. Actuators A 145 (2008) $278-282$.

[4] S.-C. Lin, W.-J. Wu, Piezoelectric micro energy harvesters based on stainless-steel substrates, Smart Mater. Struct. 22 (4) (2013) 045016.

[5] V. Ferrari, Printed thick-film piezoelectric and pyroelectric sensors, in: $M$. Prudenziati, J. Hormadaly (Eds.), Printed Films, Woodhead Publishing Limited, Sawston, 2012, pp. 221-258.

[6] G. Han, J. Ryu, W.-H. Yoon, J.-J. Choi, B.-D. Hahn, D.-S. Park, Effect of film tickness on the piezoelectric properties of lead zirconate titanate thick films fabricated by aerosol deposition, J. Am. Ceram. Soc. 94 (5) (2011) 1509-1513.

[7] T. Inoue, M. Kobayashi, $\mathrm{PbTiO}_{3} / \mathrm{Pb}(\mathrm{Zr}, \mathrm{Ti}) \mathrm{O}_{3}$ sol-gel composite for ultrasonic transducer applications, Jpn. J. Appl. Phys. 53 (7S) (2014) 07KC11.

[8] R.A. Dorey, R.W. Whatmore, Electrical properties of high density PZT and PMNPT/PZT thick films produced using ComFi technology, J. Eur. Ceram. Soc. 24 (6) (2004) 1091-1094.

[9] D. Wang, M.J. Edirisinghe, R.A. Dorey, Formation of PZT crack-free thick films by electrohydrodynamic atomization deposition, J. Eur. Ceram. Soc. 28 (14) (2008) 2739-2745.

[10]D. Wang, S.A. Rocks, R.A. Dorey, Electrohydrodynamic atomization deposition of PZT sol-gel slurry and sol infiltration on the films, J. Eur. Ceram. Soc. 32 (8) (2012) 1651-1658.

[11]D. Wang, S.A. Rocks, R.A. Dorey, Micromoulding of PZT film structures using 
electrohydrodynamic atomization mould filling, J. Eur. Ceram. Soc. 29 (6) (2009) 1147-1155.

[12]D. Wang, X. Zhu, J. Liang, T. Ren, W. Zha, W. Dong, S.A. Rocks, R.A. Dorey, Z. $\mathrm{Xu}, \mathrm{X}$. Wang, Electrohydrodynamic jet printing of PZT thick film micro-scale structures, J. Eur. Ceram. Soc. 35 (13) (2015) 3475-3483.

[13]D. Sun, S.A. Rocks, M. Edirisinghe, R.A. Dorey, Y. Wang, Electrohydrodynamic deposition of nanostructured lead zirconate titanate, J. Nanosci. Nanotechno. 5 (11) (2005) 1846-1851.

[14] A. Nithyanandan, S. Mahalingam, J. Huang, S. Rehman, E. Draper, M. Edirisinghe, Template-assisted electrohydrodynamic atomization of polycaprolactone for orthopedic patterning applications, Mater. Sci. Eng. C 33 (8) (2013) 4608-4615.

[15]H. Ghanbar, C. Luo, P. Bakhshi, R. Day, M. Edirisinghe, Preparation of porous microsphere-scaffolds by electrohydrodynamic forming and thermally induced phase separation, Mater. Sci. Eng. C 33 (5) (2013) 2488-2498.

[16]S.E. Park, S. Kim, D. Y. Lee, E. Kim, J. Hwang, Fabrication of silver nanowire transparent electrodes using electrohydrodynamic spray deposition for flexible organic solar cells, J. Mater. Chem. A 1 (45) (2013) 14286-14293.

[17]C. Luo, S. Loh, E. Stride, M. Edirisinghe, Electrospraying and electrospinning of chocolate suspensions, Food Bioprocess Tech. 5 (6) (2012) 2285-2300.

[18]G. Han, J. Ryu, W.-H. Yoon, J.-J. Choi, B.-D. Hahn, J.-W. Kim, D.-S. Park, S. Priya, Experimental investigation on the effect of top electrode diameter in PZT thick films, Mater. Lett. 65 (14) (2011) 2193-2196.

[19]L. Kerkache, A. Layadi, E. Dogheche, D. Remiens, Structural, ferroelectric and dielectric properties of $\mathrm{In}_{2} \mathrm{O}_{3}: \mathrm{Sn}$ (ITO) on $\mathrm{PbZr}_{0.53} \mathrm{Ti}_{0.47} \mathrm{O}_{3}(\mathrm{PZT}) / \mathrm{Pt}$ and annealing effect, J. Alloy Compd. 509 (20) (2011) 6072-6076.

[20]D. Kuscer, O. Noshchenko, H. Uršič, B. Malič, Piezoelectric properties of ink-jetprinted lead zirconate titanate thick films confirmed by piezoresponse force microscopy, J. Am. Ceram. Soc. 96 (9) (2013) 2714-2717.

[21]R.N. Torah, S.P. Beeby, N.M. White, Experimental investigation into the effect of 
substrate clamping on the piezoelectric behaviour of thick-film PZT elements, J. Phys. D: Appl. Phys. 37 (7) (2004) 1074-1078.

[22]Y. Liu, K.H. Lam, K. Kirk Shung, J. Li, Q. Zhou, Enhanced piezoelectric performance of composite sol-gel thick films evaluated using piezoresponse force microscopy, J. Appl. Phys. 113 (18) (2013) 187205.

[23]M.M. Carroll, A.C. Holt, Static and dynamic pore-collapse relations for ductile porous materials, J. Appl. Phys. 43 (4) (1972) 1626-1636.

[24]J.W. Lee, C.S. Park, M. Kim, H.E. Kim, Effects of residual stress on the electrical properties of PZT films, J. Am. Ceram. Soc. 90 (4) (2007) 1077-1080.

[25]T. Miyoshi, M. Nakajima, H. Funakubo, Effects of substrate clamping on electrical properties of polycrystalline piezoelectric films, Jpn. J. Appl. Phys. 48 (9) (2009) 09KD09.

[26]Meggitt PLC, https://www.meggitt.com (accessed 28 August 2017). 


\section{List of figure captions}

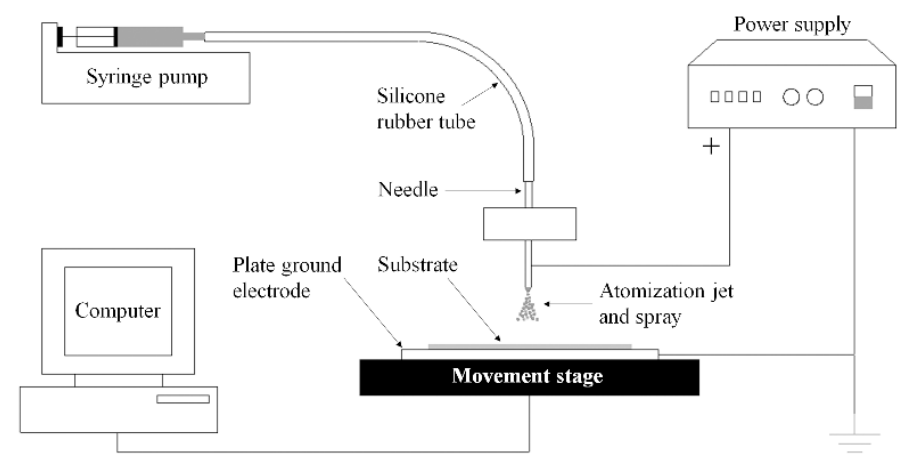

Fig. 1. Schematic diagram of E-Jet deposition equipment.

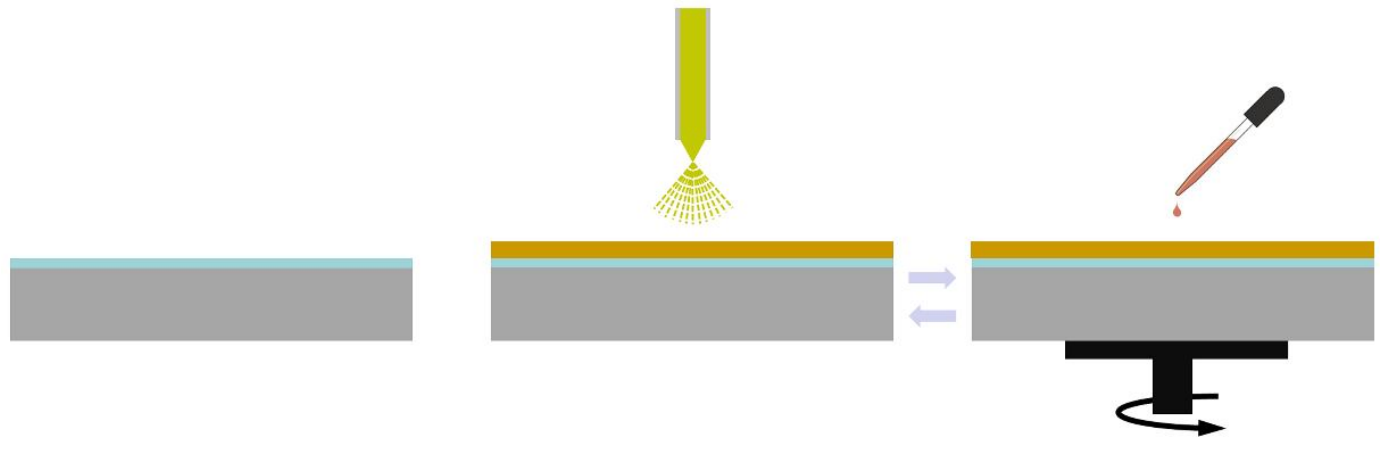

(a) Sputtering Ti/Pt bottom electrode

(b) Depositing and sol infiltrating PZT thick film

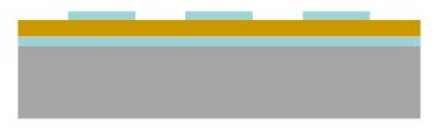

(c) Patterning Ti/Pt top electrode

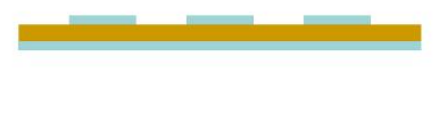

(d) Etching Si substrate

$\square \mathrm{Si} \quad \square \mathrm{Ti} / \mathrm{Pt} \quad \square \mathrm{PZT}$ slurry $\quad$ PZT sol $\quad$ PZT thick film

Fig. 2. Schematic illustrating the fabrication route of the substrate-free PZT thick films. 


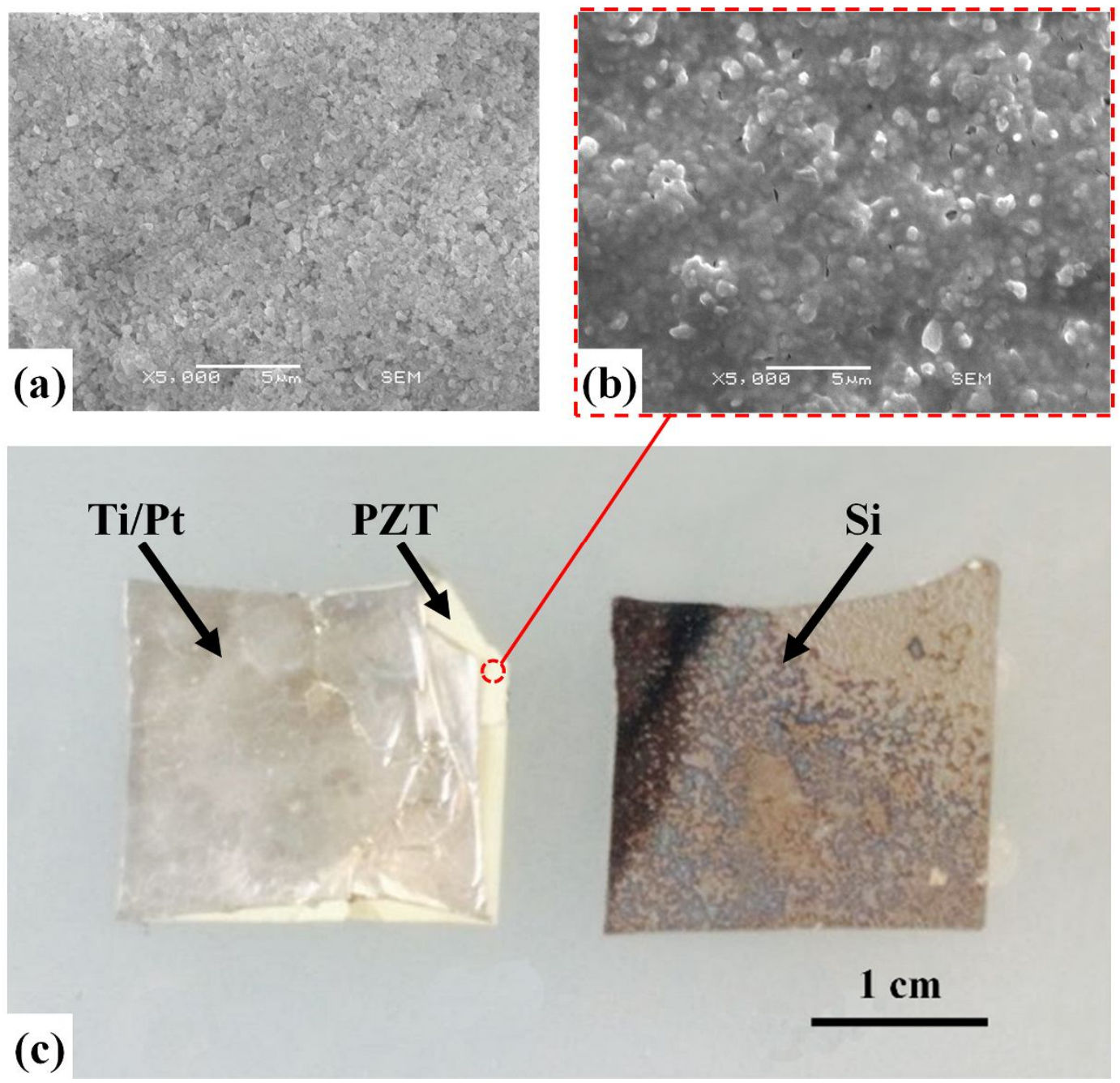

Fig. 3. Scanning electron microscope micrographs showing the microstructure of the surface of the PZT films without (a) and with (b) sol infiltration process. Photograph of the substrate-free PZT thick film and the Si substrate (c).

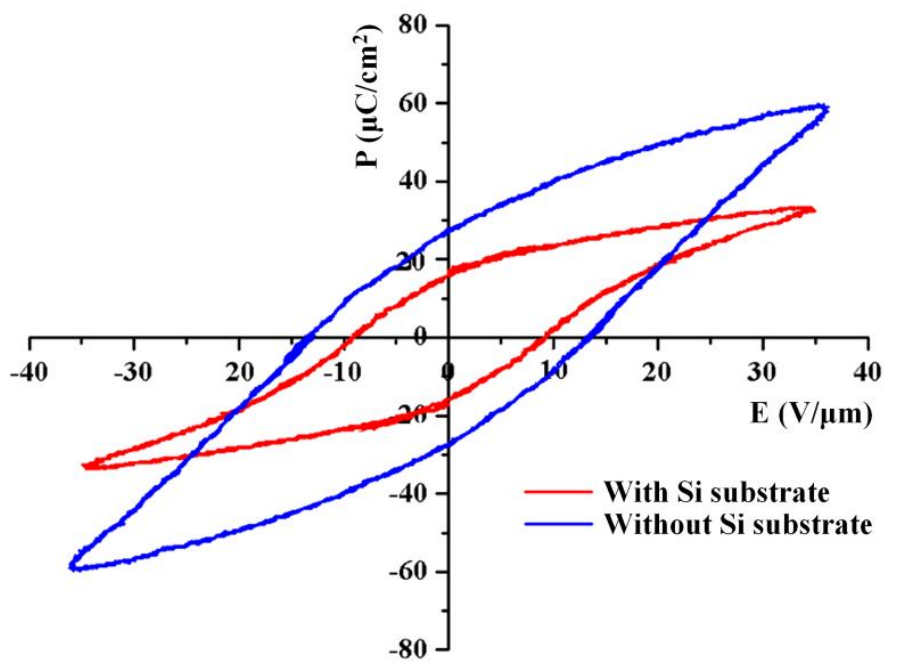

Fig. 4. Ferroelectric hysteresis loops of the PZT thick films with and without Si substrate. 


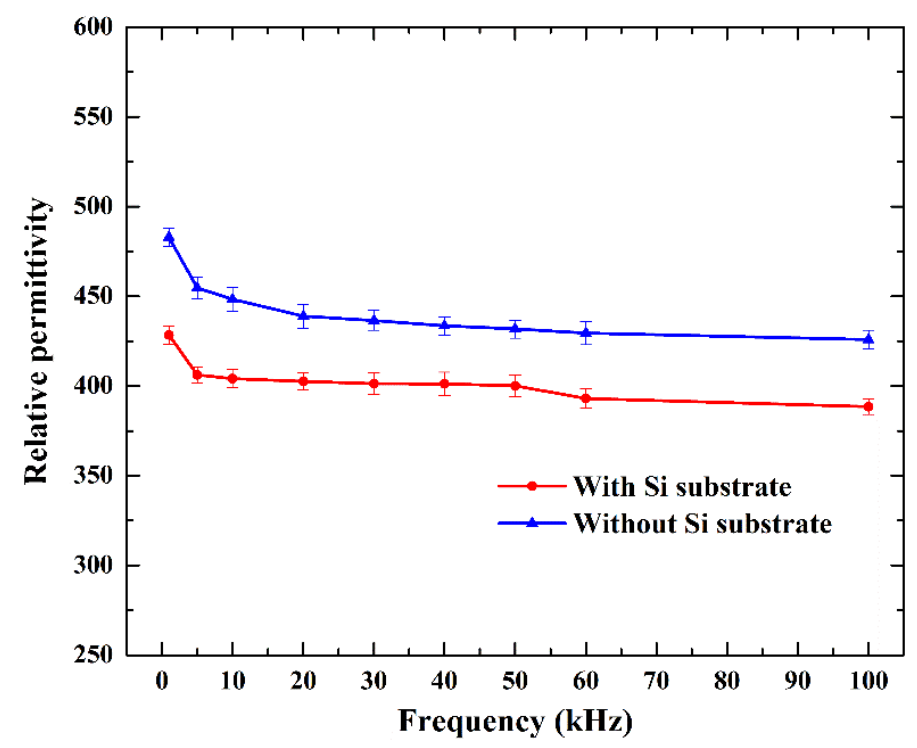

Fig. 5. Relative permittivities $\left(\varepsilon_{\mathrm{r}}\right)$ of PZT thick films with and without Si substrate in the frequency range from 1 to $100 \mathrm{kHz}$.

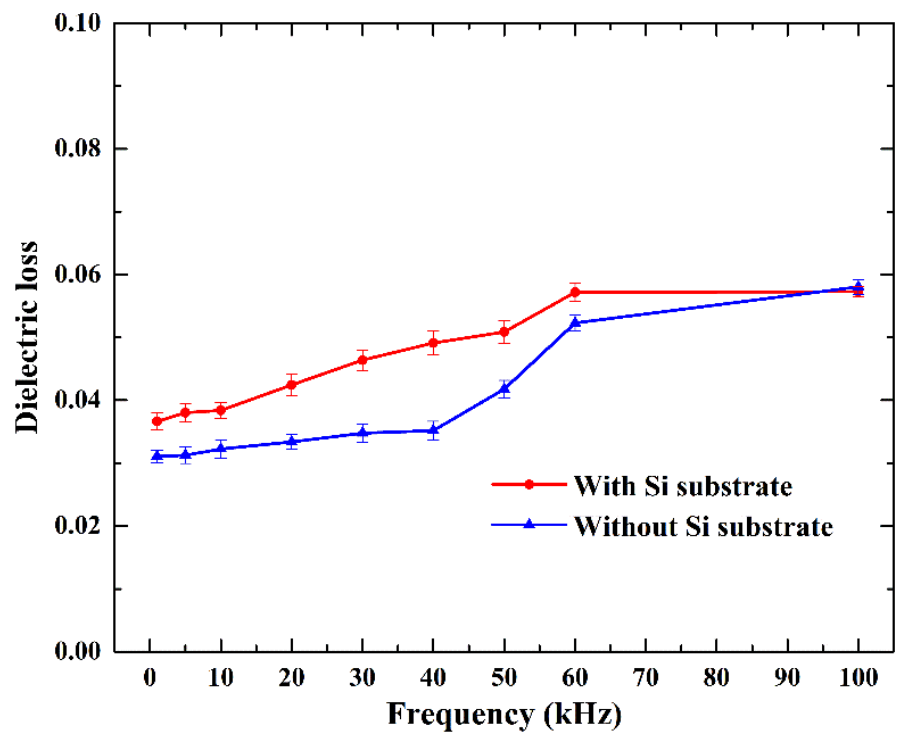

Fig. 6. Dielectric losses of PZT thick films with and without Si substrate in the frequency range from 1 to $100 \mathrm{kHz}$. 

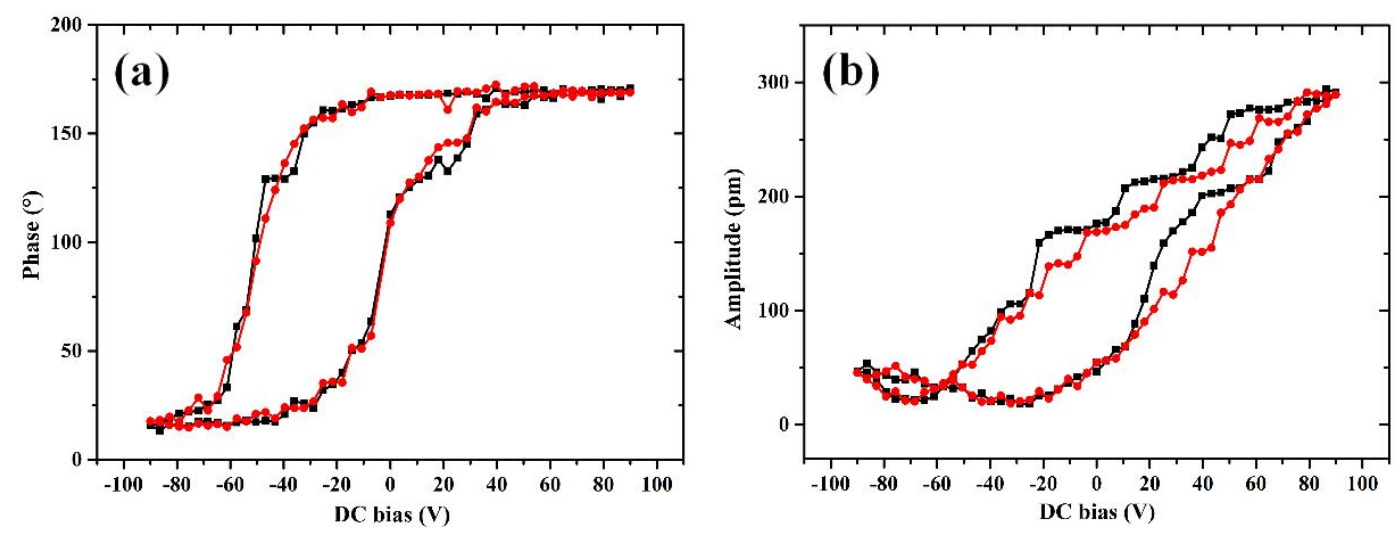

Fig. 7. Phase-voltage hysteresis loop (a) and amplitude-voltage butterfly loop (b) of the PZT thick film with Si substrate.
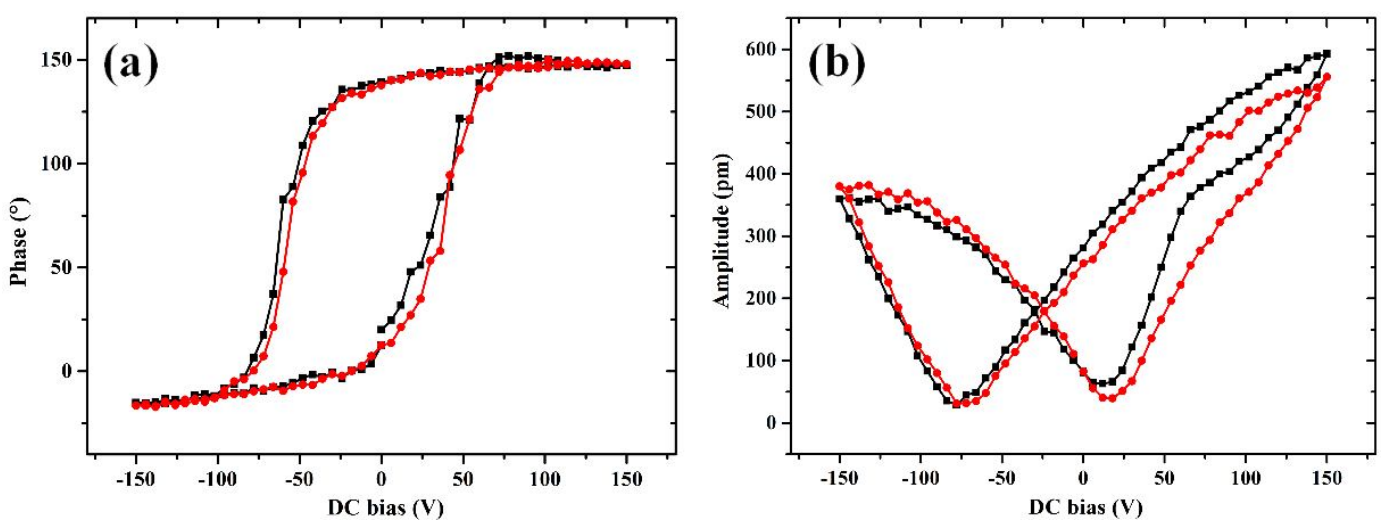

Fig. 8. Phase-voltage hysteresis loop (a) and amplitude-voltage butterfly loop (b) of the PZT thick film without Si substrate.

Fig. 9. The notation of crystal axes with polarization direction on the PZT thick film. 